\title{
Clinical Study \\ Double Lumen Tubes: Usage and Performance by Frequent and Infrequent Users
}

\author{
Sachin Jha ${ }^{1}$ and Jesse M. Ehrenfeld ${ }^{2}$ \\ ${ }^{1}$ Eastern Virginia Medical School, Norfolk, VA 23507, USA \\ ${ }^{2}$ Departments of Anesthesiology and Biomedical Informatics, Vanderbilt University Medical School, Nashville, TN 37232, USA
}

Correspondence should be addressed to Jesse M. Ehrenfeld, jesse.ehrenfeld@vanderbilt.edu

Received 10 August 2011; Accepted 5 September 2011

Academic Editors: M. Şentürk and S. J. Verbrugge

Copyright ( $\odot 2011$ S. Jha and J. M. Ehrenfeld. This is an open access article distributed under the Creative Commons Attribution License, which permits unrestricted use, distribution, and reproduction in any medium, provided the original work is properly cited.

Double lumen tubes (DLT) are commonly used to achieve lung isolation (LI). Not all anesthesiologists are frequent DLT users. Our thoracic surgical service is covered by sub-specialty anesthesiologists who are frequent DLT users. Thus, we are in a position to evaluate the performance of infrequent DLT users relative to frequent DLT users. Using statistical methods, we examined the incidence, duration and severity of hypoxia, hypercapnea and high airway pressures for patients receiving LI via DLTs placed by infrequent versus frequent users. The incidence of low $\mathrm{SpO}_{2}$, high $\mathrm{EtCO}_{2}$, or high PIP was not different between frequent and infrequent DLT users. However, when these events do occur, they are more severe (elevated $\mathrm{EtCO}_{2}$ duration, lower $\mathrm{SpO}_{2}$, higher $\mathrm{EtCO}_{2}$, higher airway pressure) among infrequent than frequent DLT users. The practical significance of these differences, which are small, is unproven. However, when episodes of hypercapnea do occur, they last much longer among infrequent than frequent DLT users.

\section{Introduction}

Double lumen tubes (DLTs) are commonly used to achieve lung isolation (LI) [1]. Placement and maintenance of DLTs is part of the subspecialty education in thoracic anesthesiology, requiring special skills, particularly fiberoptic bronchoscopy [2].

Not all anesthesiologists place DLTs frequently in their daily practice, and many large anesthesia practices maintain specialty teams with expertise in anesthesia for thoracic surgery, with DLT management being a core competency. This approach works well as long as all patients needing LI can be assigned to rooms covered by a thoracic anesthesiologist. Periodically it is impossible to meet this goal, and anesthesiologists who do not encounter DLTs in their daily practice may anesthetize patients needing LI. Existing literature concerning lung isolation studies evaluate thoracic anesthesiologists or those who are infrequent DLT users and fail to compared infrequent users [3].

In our institution, patients needing LI are periodically booked in general ORs. Our practice is for the anesthesia teams assigned to ORs booked for cases involving LI to place the DLT, even if they use these tubes infrequently. We also have a busy thoracic surgical service covered by subspecialty anesthesiologists who are frequent DLT users. Thus, we are in a position to evaluate the performance of infrequent DLT users relative to a cohort of frequent DLT user peers.

We reasoned that evidence of performance differences for DLT placement and maintenance would manifest as differences in the incidence and duration of hypoxemia, hypercapnea, and high airway pressures during clinical care. Our department uses an anesthesia information management system (AIMS). Thus, performance differences, if any, between frequent and infrequent DLT users would be present in the anesthesia record. We anticipated that departures from desirable norms would be captured by monitors and automatically stored by our AIMS, even if anesthesiologists quickly corrected the problem giving the out-of-norm value. Abnormalities in any of these variables may signify difficulties during LI that may be attributable to the management of the DLT. 
In our study, we sought differences in performance between frequent and infrequent DLT users by examining the incidence and duration of hypoxia, hypercapnea, and high airway pressures. Using these criteria, we hypothesized that infrequent DLT users will demonstrate less favorable clinical results when compared to frequent DLT users. We tested our hypothesis by retrospectively examining the performance characteristics of a large number of endotracheal DLTs inserted and maintained by both frequent and infrequent DLT users.

\section{Methods}

The Massachusetts General Hospital Human Research Committee (Massachusetts General Hospital, Boston, MA) approved this study. We conducted a retrospective review of all patients who underwent DLT placement by our thoracic anesthesia service between April 15, 2003, and December 31, 2004. (Note: a subset of the patients included in the present study ( $n=198$ of the 899 subjects) were previously reported in [1]).

Data were obtained by querying the MGH Department of Anesthesia, Critical Care and Pain Medicine's AIMS database. The AIMS contains a computer database of every anesthetic administered since the inception of the system at our institution and automatically records oxygen saturation $\left(\mathrm{SpO}_{2}\right)$, end-tidal $\left(\mathrm{EtCO}_{2}\right)$, and peak inspiratory pressure (PIP) every 30 seconds for each case. DLT placement is documented from a structured list of comments pertaining to DLT placement and via menu choices that force recording of left versus right-sided tube use. Side of surgery was also obtained. The study population included all surgical patients in the OR whose data was recorded using the AIMS database and who received right-or left-sided endobronchial intubation. The data extracted from the AIMS included the case date and ID (a unique number assigned to each case), hospital assigned medical record number, patient age, sex and ASA physical status classification, OR number, surgical procedure performed, DLT side and size, all text comments pertaining to airway management, and finally $\mathrm{SpO}_{2}, \mathrm{EtCO}_{2}$, and airway pressure data for the entire duration of the case.

Case ID data were recorded to ensure that each case identified was unique. The case descriptions were used to select only cases of chest wall, lung, pleural, esophageal and gastric surgery involving LI. All such cases were included in the analysis, and this created a group of comparable cases for which infrequent DLT users gave a significant fraction of the anesthetics.

In order to obtain the data required for this study, a query was developed to examine the physiologic monitoring data in each case where a DLT comment was recorded. We then identified the subset of LI cases within the larger AIMS database and used software rules to decide which portions of physiologic monitoring during the case corresponded to DLT facilitated LI. The physiologic data was examined from the timestamp entry of any comment pertaining to DLT placement to the timestamp associated with any of the following comments: reinstitution of bilateral ventilation, end of surgery, or extubation. If none of these comments were present in the AIMS, we examined the physiologic data until the patient departed from the OR.

We estimated the fraction of DLT cases captured by our query by selecting a uniform sample of days from the AIMS DLT results. We then manually searched the OR schedule and medical records for these days for any additional DLT cases not flagged by triggering comments in the AIMS data. We also performed a manual comparison of the data extracted by our query with the anesthesia records from $5 \%$ of the cases randomly selected from the study group to ensure the accuracy of our data.

The identities of individual anesthesia providers were not recorded. Instead, DLTs placed in the ORs utilized by the thoracic anesthesia service were deemed to have been placed by a frequent user. All other DLT placements were deemed to have been performed by infrequent users. Attending anesthesiologists who work on the thoracic anesthesia service (frequent users) were accustomed to placing DLTs daily, whereas the attending physicians on other services (infrequent users) only occasionally place DLTs (typically $<1$ per month).

Once the AIMS data query had been performed, we compared the occurrence of frequent versus infrequent DLT users among our anesthesiologists. Next, we examined the physiologic data to determine the incidence, duration, and severity of episodes of hypoxemia. For each DLT placement, we tabulated the incidence, duration and severity of hypoxia $\left(\mathrm{SpO}_{2}<90 \%\right)$, hypercapnea $\left(\mathrm{EtCO}_{2}>45 \mathrm{mmHg}\right)$, and high airway pressures ( $\mathrm{PIP}>35 \mathrm{~cm} \mathrm{H}_{2} \mathrm{O}$ ) between the start and end of LI. For an episode of hypoxemia, hypercapnea, or high airway pressure to be included in our tabulation, it needed to last a minimum of $180 \mathrm{~s} \mathrm{(} 3 \mathrm{~min})$. These criteria were chosen in advance of performing the data queries.

The data were tabulated in Microsoft Excel (Redmond, WA), and statistical analysis was performed using the JMP statistical software package (Cary, NC). All comparisons of DLT performance were planned. We compared continuous data using Student's $t$-test. Categorical data were compared using $X^{2}$ tests. In all tests of significance, a $P$ value of $<0.05$ was considered to be significant.

\section{Results}

We found 889 cases having LI with a comment pertaining to DLT placement that would have flagged the case for inclusion in our dataset. We were further able to divide the data set into cases performed by frequent users $(n=790)$ and cases performed by infrequent users $(n=99)$ (Table 1). Cases managed by infrequent users were managed by a total of 84 different attending anesthesiologists who each managed on average 1.2 DLT cases per year.

Data collection for $\mathrm{SpO}_{2}, \mathrm{EtCO}_{2}$, and airway pressures ran from the timestamp entry of any comment pertaining to DLT placement to the timestamp associated with any of the following stopping conditions: reinstitution of bilateral ventilation ( $62 \%$ of cases), end of surgery ( $21 \%$ of cases), extubation ( $1 \%$ of cases), or until the patient departed the OR ( $16 \%$ of cases). 
TABLE 1: Characteristics of patients in frequent and infrequent double lumen tube user groups.

\begin{tabular}{lccc}
\hline & $\begin{array}{c}\text { Frequent } \\
(n=790)\end{array}$ & $\begin{array}{c}\text { Infrequent } \\
(n=99)\end{array}$ & $P$ \\
\hline Age $($ years $\pm \mathrm{SD})$ & $62 \pm 14$ & $49 \pm 21$ & $<0.001$ \\
Sex $(\mathrm{M} / \mathrm{F})$ & $442 / 349$ & $66 / 33$ & $<0.05$ \\
ASA PS $(1 / 2 / 3 / 4)$ & $13 / 408 / 346 / 24$ & $15 / 41 / 36 / 7$ & $<0.005$ \\
\hline
\end{tabular}

Values for continuous data are reported as mean \pm standard deviation and compared by $t$ testing. Categorical data were compared by $X^{2}$ analysis. Age is reported as mean \pm standard deviation. ASA physical status is reported as the number of patients in each category.

ASA PS: American Society of Anesthesiologists physical status classification.

The ASA physical status classifications, age and sex distributions for patients in the frequent and infrequent PLT user groups are shown in Table 1 . The two groups are not comparable with respect to sex ratio, age, and ASA physical status classification. However, with respect to each variable, the patient characteristics in the infrequent DLT user group favor better outcome variable performance. In other words, the patients in the infrequent DLT user group were younger and had less comorbidity as assessed by ASA PS.

Table 2 shows results for a large number of cases under real-world conditions (routine, urgent, and emergent utilization of these tubes). The incidence of low $\mathrm{SpO}_{2}$, high $\mathrm{EtCO}_{2}$, or high PIP was not different between frequent and infrequent DLT users. However, when these events do occur, they are more severe (lower $\mathrm{SpO}_{2}$, higher $\mathrm{EtCO}_{2}$, higher airway pressure) among infrequent than frequent DLT users. Additionally, $\mathrm{EtCO}_{2}$ elevations persist for much longer among infrequent DLT users. Given the retrospective nature of our study, we do not have data regarding the specific treatment for these episodes of hypercarbia.

\section{Discussion}

We used an AIMS database to perform a single-center retrospective study comparing the intraoperative performance of frequent and infrequent DLT users during LI. From Table 1, it is apparent that despite attempts to establish a homogeneous population by excluding specialty case types, the patient groups have significant demographic differences. Patients in the infrequent DLT user group were younger, more likely to be male, and more likely to be at an extreme of the ASA physical status scale. Thus, any conclusions drawn from the performance data must be considered in light of these differences.

There are also potential limitations with respect to the case finding strategy. For example, we may have missed cases in which a DLT was employed but for which no DLT comment was entered into the AIMS. If we assume that this omission was equally likely to have occurred in both groups, we would expect this to be equally distributed and hence unlikely to bias our results-even if DLT use was under reported.
As an academic institution, residents were involved in these cases. While the attending anesthesiologists were not continually present in the operating room, they were immediately available to their residents. It is common practice to encourage independent, prudent, and active management of DLTs and lung isolation by the residents. This includes the continued performance of diagnostic and troubleshooting maneuvers during the intraoperative period such as lung auscultation, fiberoptic bronchscopy, and continuous evaluation of airway pressures, tidal volumes, and overall ventilation. Frequent bronchoscopic examinations and tube placement adjustments are encouraged with or without direct supervision of the attending anesthesiologist. Assuming the incidence of resident involvement was equivalent in both data sets, any effect that may have made on the study can be assumed to have occurred equally in both data sets.

There were no additions or departures of surgeons involved in these cases during the course of the study. However, as this is a teaching hospital, surgical residents were involved in almost every case and that group of providers did change over time. Additionally, our study was limited to a single institution and thus our results may not be consistent with other institutions.

A final criticism of retrospective studies is the strong possibility of selection bias influencing the result. We attempted to protect against this bias by including all cases that met our predetermined inclusion criteria of all DLT placements for case types performed both on our thoracic anesthesia service and in the general surgical service. There is also a possibility that preconceptions will influence the way cases are identified and the way data are handled; however, the use of automatic data collection with an AIMS helps protect against such bias. We used an automatic query of the entire cohort of patients to obtain the performance data, rather than a manual case-finding strategy, which again protects against selection bias. Furthermore, the data are derived from routine clinical practice, giving our result realworld validity by eliminating potential biases introduced by observation.

This is a potential strength of the methodology in that our primary data were collected automatically and uniformly for a large number of DLT cases by the AIMS. Multiple prior investigations indicate that automated records have less missing data and are better reflections of monitor output than hand-written records $[4,5]$. This is particularly true during busy periods, such as would be the case if the anesthesiologists were attending to a problem with the DLT. AIMS also records artifacts, but any errors due to artifact would be uniformly distributed between frequent and infrequent users. Furthermore, the data are derived from routine clinical practice, giving our result real-world validity by eliminating potential biases introduced by observation.

Overall, we were unable to detect a difference in the incidence of low $\mathrm{SpO}_{2}$, high $\mathrm{EtCO}_{2}$, or high airway pressures between frequent and infrequent DLT users. Although the incidence of problems related to DLTs is the same between groups, the severity of each problem is greater for infrequent users. The practical significance of these differences in severity, which are small, remains to be determined. 
TABLE 2: Hypoxia, hypercarbia, and high airway pressures in patients of frequent and infrequent double lumen tube users.

\begin{tabular}{lccc}
\hline & Frequent $(n=790)$ & Infrequent $(n=99)$ & $P$ \\
\hline Cases with $\mathrm{SpO}_{2}<90 \%$ & 79 & 14 & 0.339 \\
Mean duration $\pm \mathrm{SD}(\mathrm{min})$ & $7.4 \pm 5.6$ & $7.0 \pm 4.3$ & 0.493 \\
Mean lowest $\mathrm{SpO}_{2} \pm \mathrm{SD}(\%)$ & $85 \pm 4$ & $82 \pm 6$ & $<3$ \\
Cases with $\mathrm{EtCO}_{2}>45 \mathrm{mmHg}$ & 217 & $15.1 \pm 20.0$ & 0.348 \\
Mean duration $\pm \mathrm{SD}(\mathrm{min})$ & $9.3 \pm 9.4$ & $54 \pm 6$ & $<05$ \\
Mean highest $\mathrm{EtCO} \pm \mathrm{SD}$ & $51 \pm 5$ & 57 & $<0.01$ \\
Cases with $\mathrm{PIP}>35 \mathrm{~cm} \mathrm{H} 2 \mathrm{O}$ & 552 & $22.3 \pm 22.6$ & 0.308 \\
Mean duration $\pm \mathrm{SD}(\mathrm{min})$ & $18.4 \pm 18.7$ & $45 \pm 7$ & 0.057 \\
Mean highest $\mathrm{PIP} \pm \mathrm{SD}$ & $43 \pm 5$ & $<0.005$ \\
\hline
\end{tabular}

Values for continuous data are reported as mean \pm standard deviation and compared by $t$ testing. Categorical data were compared by $X^{2}$ analysis. SD: standard deviation; PIP: peak inspiratory pressure.

\section{Conclusion}

There are differences between frequent and infrequent DLT users with respect to the duration of hypercarbia and high airway pressures when they do occur. Hypercarbia, in particular, lasts much longer in the patients of infrequent DLT users than in the patients of frequent DLT users.

\section{References}

[1] J. M. Ehrenfeld, J. L. Walsh, and W. S. Sandberg, "Rightand left-sided mallinckrodt double-lumen tubes have identical clinical performance," Anesthesia and Analgesia, vol. 106, no. 6, pp. 1847-1852, 2008.

[2] P. D. Slinger, "Fiberoptic bronchoscopic positioning of doublelumen tubes," Journal of Cardiothoracic Anesthesia, vol. 3, no. 4, pp. 486-496, 1989.

[3] J. H. Campos, E. A. Hallam, T. Van Natta, and K. H. Kernstine, "Devices for lung isolation used by anesthesiologists with limited thoracic experience: Comparison of double-lumen endotracheal tube, and arndt wire-guided endobronchial blocker," Anesthesiology, vol. 104, no. 2, pp. 261-266, 2006.

[4] A. Eden, M. Grach, Z. Goldik et al., "The implementation of an anesthesia information management system," European Journal of Anaesthesiology, vol. 23, no. 10, pp. 882-889, 2006.

[5] J. Balust and A. MacArio, "Can anesthesia information management systems improve quality in the surgical suite?" Current Opinion in Anaesthesiology, vol. 22, no. 2, pp. 215-222, 2009. 


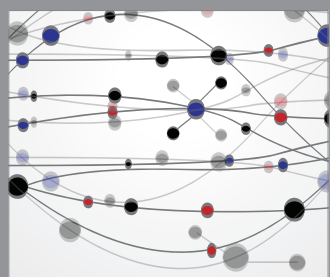

The Scientific World Journal
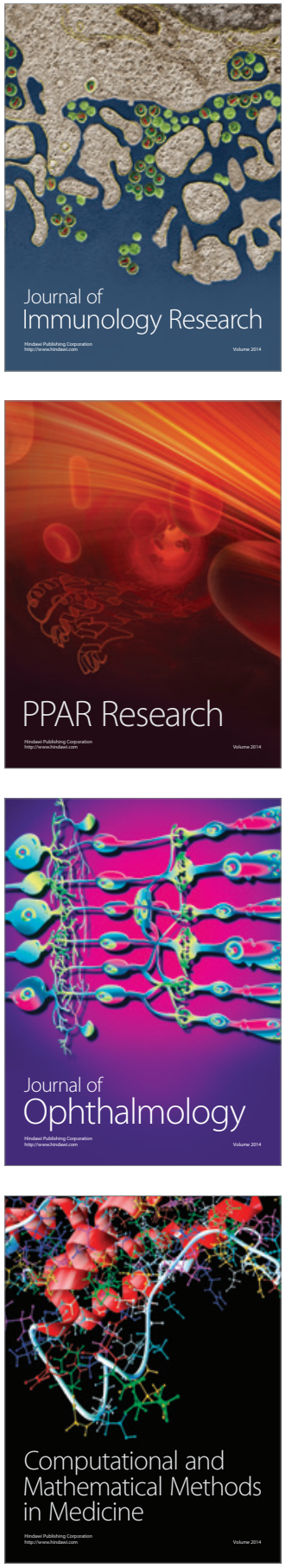

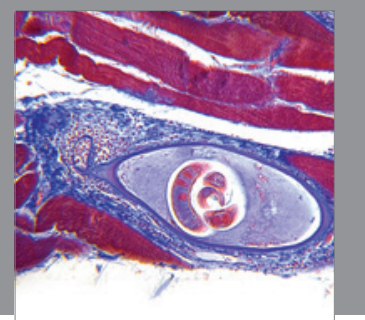

Gastroenterology

Research and Practice
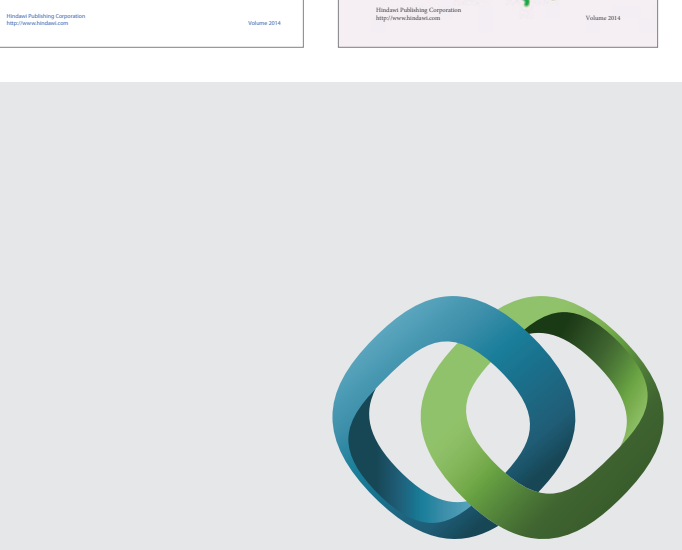

\section{Hindawi}

Submit your manuscripts at

http://www.hindawi.com
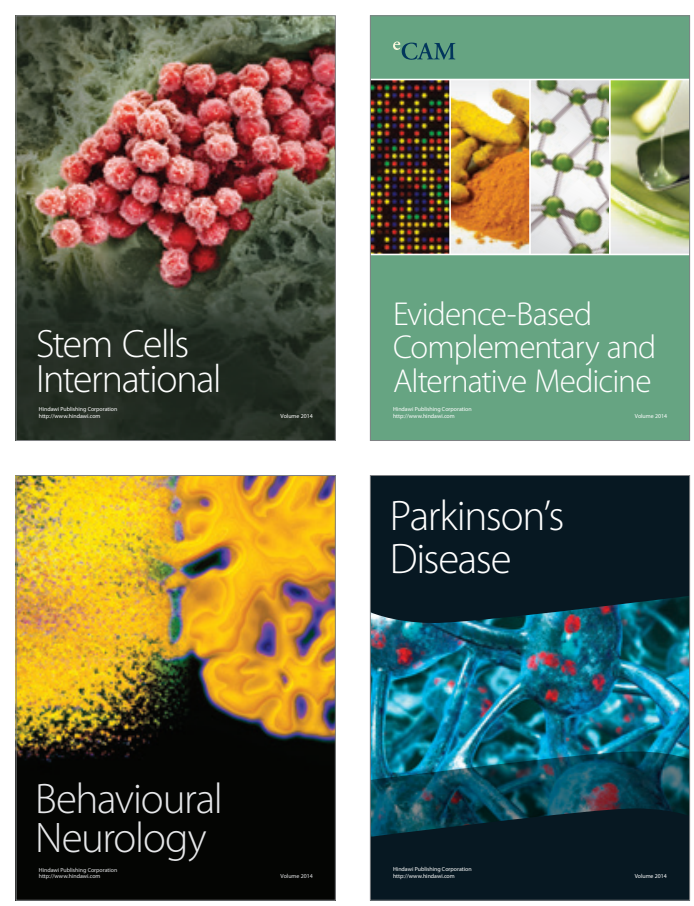

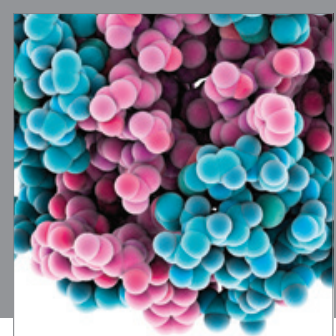

Journal of
Diabetes Research

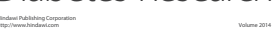

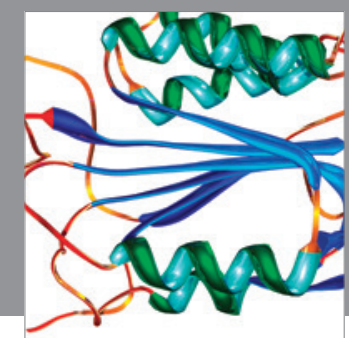

Disease Markers
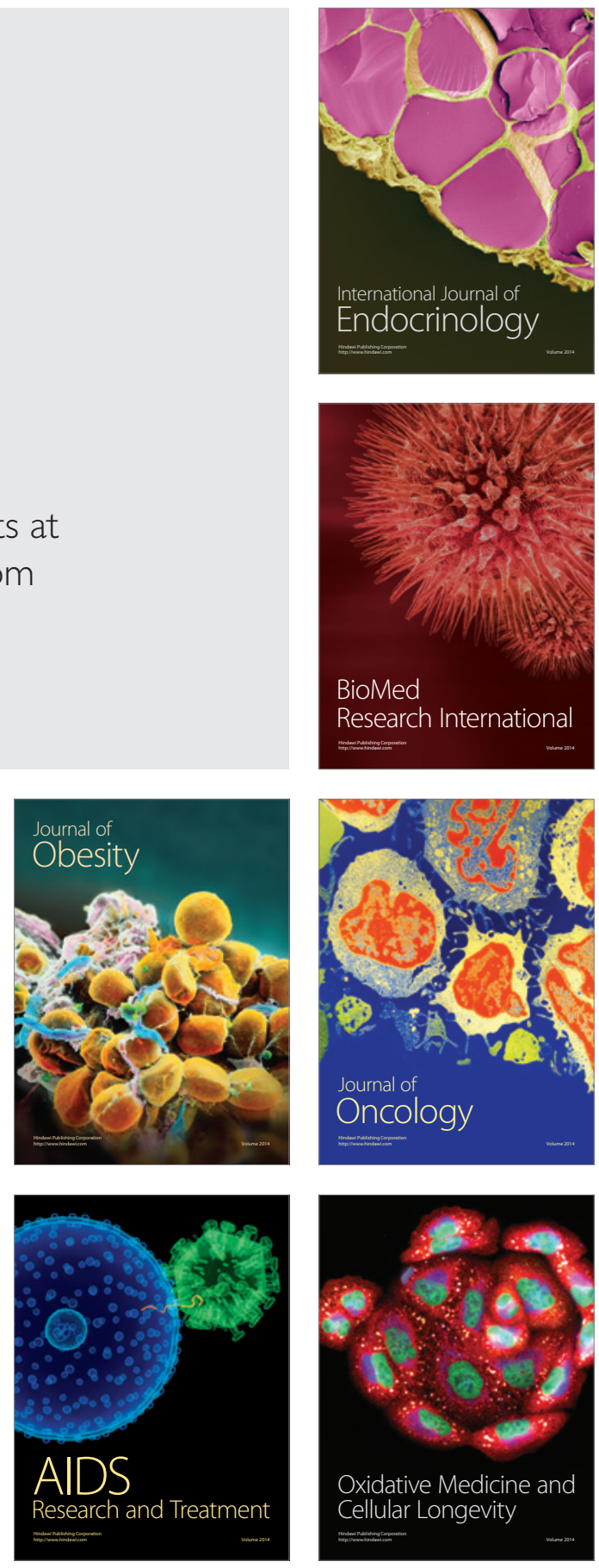